\title{
Minimally Invasive Anal Fistula Treatment—a True Minimal Invasive Surgical Procedure
}

\author{
P. K. Chowbey ${ }^{1}$
}

Received: 2 November 2015 / Accepted: 2 November 2015 / Published online: 15 December 2015

(C) Association of Surgeons of India 2015

A surgical procedure comprises of two aspects - the surgical access and surgery on the target organ. For decades, general surgeons focussed their minds to propel development in the latter, i.e., surgery on the target organ. Trauma due to surgical access was considered inevitable. Patient discomfort, debility, and disfigurement were considered to be a small price the patients were paying in the larger interest of surgical cure. The advent of minimal access surgery revolutionized the concept of surgical practice. The approach placed the patient at the center of all surgical decisions. It was for the first time that the comfort and needs of the patient were given paramount importance. Through minimal access surgery, the trauma of surgical access has been dramatically minimized, with shorter hospital stay and greater patient satisfaction. Today, minimal access surgery is firmly established in the armamentarium of surgeons worldwide.

The early postoperative sequelae following management of anal fistulae in terms of postoperative pain, need for dressings, and time taken off work has not been reported much in literature. Mostly, long-term outcomes in terms of recurrence and incontinence have been addressed in studies reported so far. Traditional techniques including fistulectomy and use of cutting seton have been associated with incontinence, especially in patients who have had previous surgery. Mucosal advancement flaps are technically challenging and are associated with high recurrence rates and high rates of incontinence postoperatively.

Although surgical field has undergone significant evolution over the past few decades, the surgical management of the

P. K. Chowbey

pradeepchowbey@gmail.com

1 New Delhi, India perianal anomalies is yet to witness a technological breakthrough. Apart from treatment of hemorrhoids, there have not been many surgical innovations for perianal infective pathologies. The newer treatment options include use of fibrin glue, bio-prosthetic plugs, and ligation of intersphincteric fistula tract (LIFT) and video-assisted anal fistula treatment (VAAFT). LIFT procedure has been associated with good healing rates. The early results with the use of VAAFT have been encouraging so far.

The video-assisted anal fistula treatment (VAAFT) was developed by Professor P. Meinero in 2006 [1]. However, we strongly feel and advocate the terminology of minimally invasive anal fistula treatment (MAFT), which is much more appropriate and is the correct description of the procedure. MAFT qualifies as a true minimal invasive surgical procedure. There are no iatrogenic incisions for access on the patient. Surgical access is obtained from a pre-existing pathological opening of the fistula. The technique comprises identification and secure internal closure of the internal fistula opening and visualization with cauterization of the fistulous tract using a specially designed fistuloscope. The MAFT is based on the same principles as other procedures for closing the internal opening and obliterating the tract with the innovation that allows precise identification of the fistula anatomy and the internal opening by fistuloscopy and fulguration of the tract walls under direct vision. This approach allows the identification and treatment of all the secondary tracts and the abscess cavities connected to the main track. In our opinion, the adoption of fistuloscope-aided exploration along with effective closure of the internal opening and reinforcing the closure of the opening from the inner side of the staple/suture line is the most effective way of achieving a high healing rate for complex anal fistulas with preservation of the anal sphincter.

We adopted the MAFT technique in April 2011 in an effort to reduce early postoperative morbidity and offer patients 
advantages of minimally invasive surgery. We have previously reported our experience with MAFT in 416 patients [2]. MAFT was performed as day care procedure in $94 \%$ patients with a very low rate of re-admission. Patients experienced minimal early and late postoperative pain and analgesic requirements in the postoperative period as indicated by their VAS scores. In our experience, the recurrence rate was $26.1 \%$ and the patients with recurrence underwent MAFT procedure again. Since the technique creates no postoperative perianal wounds, the requirement for major postoperative dressings is obviated. Small dressings are placed at the site of surgery that can be managed easily by the patients. This also ensures that patients return to work early. MAFT can be easily performed again for recurrent fistulae, and this is acceptable to patients in view of low morbidity and rapid recovery.

A new and innovative surgical technique normally involves added expenditure. The advantage of MAFT is that the fistuloscope is reusable and has low recurring expenditure. Early in our experience with MAFT, we performed MRI in all patients to identify the anatomy of fistula tract and secondary tracts. As our experience with MAFT increased, MRI was reserved for selective patients. At present, in our opinion, the technique precludes the necessity of a pre-operative radiological investigation for a fistula. The extra cost of changing daily dressings is also avoided.

The principles and concept of MAFT can be applied in the surgical management of other perianal pathological conditions like pilonidal sinuses, foreign body sinuses, and nonhealing sinuses of various etiologies. It is also possible that many of the pathologies elsewhere in the body can be treated with a similar minimal access approach that involves small incisions, drainage, and debridement/fulguration of the abscess cavity and associated infective tracks. MAFT is safe and feasible and can be mostly performed as a day care procedure. Since MAFT is a sphincter-saving and scarless procedure, it can be performed in patients with recurrence following the procedure, with minimal morbidity. There are distinct advantages to patients in terms of lesser pain, absence of perianal wounds, faster recovery, and early return to work. However, applicability of MAFT in very high extra sphincteric fistulae remains unclear at present. Long-term results from more centers are awaited, especially for fistula recurrence rates.

\section{References}

1. Meinero P, Mori L (2011) Video-assisted anal fistula treatment (VAAFT): a novel sphincter-saving procedure for treating complex anal fistulas. Tech Coloproctol 15:417-422

2. Chowbey PK, Khullar R, Sharma A, Soni V, Najma K, Baijal M (2013) Minimally invasive anal fistula treatment (MAFT) - an appraisal of early results in 416 patients. Indian J Surg. doi:10.1007/ s12262-013-0977-2 